\title{
Absence of Highest-Spin Ground States in the Hubbard Model
}

\section{András Sütő ${ }^{\star}$}

Institut de Physique Théorique, Ecole Polytechnique Fédérale de Lausanne, CH-1015 Lausanne, Switzerland

Received July 23, 1990

\begin{abstract}
The Hubbard model $H=-t \Sigma c_{x \sigma}^{\dagger} c_{y \sigma}+U \Sigma n_{x \uparrow} n_{x \downarrow}$ with $N$ electrons and periodic boundary condition is studied on $v$-dimensional $L_{1} \times \cdots \times L_{v}$ lattices. It is shown that for any value of $U$ there is no ground state with maximal spin $(S=N / 2)$ in the following cases: (i) $\mathbb{Z}^{v}(v \geqq 2)$ at low electron densities; with one hole if $t>0$ and $L_{i}$ is odd for some $i$; with two holes if $t<0$, or if $t>0$ and all the $L_{i}$ are even. (ii) The bcc lattice at low densities; with two holes if $t<0$, or if $t>0$ and all the $L_{i}$ are even; with $2, \ldots, 6$ holes if $L_{i}=L$ and $t<0$, or if $t>0$ and $L$ is even. (iii) The triangular lattice at densities near 0 and 1 if $t>0$; with two holes if $t<0$; with 2,3,4 holes if $t<0$ and $L_{1}=L_{2}$. (iv) The $f c c$ lattice at densities near 0 and 1 if $t>0$; with two holes if $t<0$. Some results for the one dimensional model are also presented.
\end{abstract}

\section{Introduction}

To prove the appearance of ferromagnetism in some reasonable model of itinerant electrons has long been the obsession of theoretical and mathematical physicists. Perhaps the simplest such model is the Hubbard model. On a finite lattice $\Lambda$ it is given by the Hamiltonian

$$
H_{U}=-\sum_{x \neq y \in \Lambda} t_{x y}\left(c_{x \uparrow}^{\dagger} c_{y \uparrow}+c_{x \downarrow}^{\dagger} c_{y \downarrow}\right)+U \sum_{x \in \Lambda} n_{x \uparrow} n_{x \downarrow}=H_{0}+U H_{I} .
$$

Here $c_{x \sigma}$ and its adjoint $c_{x \sigma}^{\dagger}$ are fermion annihilation and creation operators which satisfy the anticommutation relations

$$
\begin{aligned}
& c_{x \sigma}^{\dagger} c_{y \tau}+c_{y \tau} c_{x \sigma}^{\dagger}=\delta_{x y} \delta_{\sigma \tau}, \\
& c_{x \sigma} c_{y \tau}+c_{y \tau} c_{x \sigma}=0 .
\end{aligned}
$$

\footnotetext{
* On leave from the Central Research Institute for Physics, Budapest
} 
The operator $n_{x \sigma}=c_{x \sigma}^{\dagger} c_{x \sigma}$ has eigenvalues 0 and 1 which are the possible electron occupation numbers on site $x$ with spin $\sigma . U$ and $t_{x y}=t_{y x}$ are real parameters. $H_{0}$ corresponds to the kinetic energy and the second term with $U>0$ is aimed to represent a screened Coulomb repulsion between electrons of different spins. The spin operators in terms of $c_{x \sigma}^{\dagger}$ and $c_{x \sigma}$ are expressed as

$$
S_{x}^{z}=\frac{1}{2}\left(n_{x \uparrow}-n_{x \downarrow}\right), \quad S_{x}^{+}=\left(S_{x}^{-}\right)^{\dagger}=c_{x \uparrow}^{\dagger} c_{x \downarrow} .
$$

The total spin and number operators

$$
\begin{aligned}
& S^{z}=\sum_{x \in \Lambda} S_{x}^{z}, \quad S^{ \pm}=\sum_{x \in \Lambda} S_{x}^{ \pm}, \\
& N_{\sigma}=\sum_{x \in \Lambda} n_{x \sigma}, N=N_{\uparrow}+N_{\downarrow},
\end{aligned}
$$

commute separately with $H_{0}$ and $H_{I}$.

The problem of ferromagnetism at zero temperature consists in proving that the ground state of $H_{U}$ exhibits ferromagnetic long-range order. If the number of electrons, $N$, is fixed then $H_{U}$ should have ground states which are also eigenstates of

$$
S_{o p .}^{2}=\left(S^{z}\right)^{2}+\frac{1}{2}\left(S^{+} S^{-}+S^{-} S^{+}\right)
$$

with eigenvalue $S(S+1)$, where $S=O(N)$. Rigorous works for proving or disproving such a claim are scarce. Lieb and Mattis [1] showed that in one dimension the ground state is a state with minimal spin $\left(S_{\min }=0\right.$ or $\left.1 / 2\right)$ if $U<\infty$. Lieb recently proved [2] that independently of the lattice structure, for $N$ even the ground state is unique (and hence has $S=0$ ) if $U<0$. For $U>0$ at half-filling $(N=|\Lambda|)$ on bipartite lattices $\left(\Lambda=A \cup B, A \cap B=\emptyset\right.$ and $t_{x y}=0$ unless $x \in A$ and $y \in B$ or vice versa) Lieb [2] obtains that the ground state is a $(2 S+1)$-fold degenerate state with $S=(1 / 2)|| B|-| A||$. If $|B| \neq|A|$ then $S=O(N)$ is possible, although the resulting order is probably antiferromagnetic and thus the ground state is ferrimagnetic. The only genuine example of ground state ferromagnetism is due to Nagaoka [3], who proves that on $v>1$ dimensional lattices with $t_{x y}<0$ and for $N=|\Lambda|-1$, the ground state is an $(N+1)$-fold degenerate saturated ferromagnetic state (i.e., it has $S=N / 2$, the maximal possible value for $N$ electrons). This result is obtained for $U=\infty$ but by continuity it extends to large but finite $U$ (see Appendix). A recent easy proof is due to Tasaki [4].

The $U=\infty$ limit in one dimension is somewhat special. It is commonly believed that there are ground states with all possible values of $S$. In fact, this holds true for free boundary condition (combine results of [1] and of Aizenman and Lieb [5] or see the Appendix). In the periodic case there may or may not be ground states with $S=N / 2$ depending on the sign of $t_{x y}$ and the parity of $N$ ([5] and present paper). By now, there exists a full description of the ground states and the thermodynamics [6] which shows among others that the average ground state magnetisation is $O\left(N^{1 / 2}\right)$.

In the present paper I prove the absence of saturated ferromagnetism in certain cases, i.e., that any ground state of $H_{U}$ has $S<N / 2$. The highest-spin subspace $(S=N / 2)$ plays a particular role in the Hubbard model. Since each doubly occupied site reduces $S$ by one unit and since $H_{I}$ counts the doubly occupied sites, $H_{I} \psi=0$ for any state $\psi$ with $S=N / 2$. The eigenstates and eigenvalues of $H_{U}$ in the subspace $S=N / 2$ are those of $H_{0}$ in the same subspace. Let $|F\rangle$ denote the lowest lying 
state of $H_{U}$ in the subspace $S=S^{z}=N / 2$. To show that $|F\rangle$ is not a ground state of $H_{U}$ for any $U$, it suffices to find a state with no doubly occupied site the energy of which is below that of $|F\rangle$. In a recent paper, Shastry, Krishnamurthy and Anderson (SKA [7]) propose a family of trial states, originally invented by Roth [8], and claim that the minimal energy within this family is below the energy of $|F\rangle$ if the electron concentration $\rho=N /|\Lambda|$ is sufficiently low. The following theorem is a rigorous version of SKA's assertion.

Recall that a Bravais lattice in $v$ dimensions is a subgroup of $\mathbb{R}^{v}$ of the form

$$
\mathbb{L}=\left\{n_{1} a_{1}+\cdots+n_{v} a_{v} \mid n_{i} \in \mathbb{Z}\right\},
$$

where the primitive vectors $a_{1}, \ldots, a_{v} \in \mathbb{R}^{v}$ are linearly independent. The point group of $\mathbb{L}$ is the maximal subgroup of $O_{v}(\mathbb{R})$ which leaves $\mathbb{L}$ invariant. $\mathbb{L}$ will be called $v$-gonal if the primitive vectors can be chosen so that for $i=1, \ldots, v$,

$$
\left|a_{i}\right|=a_{0}=\min \{|x| \mid x \in \mathbb{L}, x \neq 0\}
$$

and all the vectors in $\mathbb{L}$ of length $a_{0}$ are related by point group symmetry. Throughout the paper we consider finite parts $\Lambda$ of $v$-gonal lattices with periodic boundary condition,

$$
\Lambda=\mathbb{L} /\left\{L_{1} a_{1}, \ldots, L_{v} a_{v}\right\},
$$

and a constant nearest-neighbor hopping

$$
t_{x y}=t_{x-y}=t \neq 0 \quad \text { if } \quad|x-y|=a_{0} .
$$

In a generic $v$-gonal lattice there are $2 v$ nearest neighbors, thus $H_{U}$ with (1.8) is nothing else than $H_{U}$ with (1.8) on $\mathbb{Z}^{\nu}$. So in two and three dimensions the $v$-gonal lattices with constant nearest- neighbor hopping will be the square, triangular, $s c, f c c$ and $b c c$ lattices.

The number of electrons is fixed,

$$
N<|\Lambda| \text {. }
$$

We use the short-hand $H_{U \leqq \infty}$ for " $H_{U}$ for any $U \leqq \infty$." $H_{\infty}$ is defined in the Appendix. $E_{\Lambda, N}(M)$ denotes the smallest eigenvalue of $H_{U}$ for $N$ electrons in the subspace $S=S^{z}=M . L_{\min }$ and $L_{\max }$ stand for the minimum and maximum of $L_{1}, \ldots, L_{v}$, respectively.

Theorem 1 (SKA). Let $\mathbb{L}$ be any v-gonal lattice in $v \geqq 2$ dimensions. For $\mathbb{Z}^{v}$ and the bcc lattice the sign of $t$ is arbitrary; for the other lattices (as triangular or fcc) let $t>0$. There exists $a \rho_{0}>0$ and positive functions $c(\rho)$ and $L_{0}(\rho)$, depending on the lattice, such that for any $\rho \in\left(0, \rho_{0} / 2\right), H_{U \leqq \infty}$ has no ground state with $S=N / 2$ and

$$
E_{\Lambda, N}(N / 2)-E_{\Lambda, N}(N / 2-1)>|t| c(\rho)
$$

if $\rho \leqq N /|\Lambda| \leqq \rho_{0}-\rho$ and $L_{\min }>L_{0}(\rho)$.

For the triangular and fcc lattices there exists also a $\rho_{1}, \rho_{0} \leqq \rho_{1}<1$, such that for any $\rho \in\left(0,\left(1-\rho_{1}\right) / 2\right)$ the same results hold if $\rho_{1}+\rho \leqq N /|\Lambda| \leqq 1-\rho$ and $L_{\min }>L_{0}(\rho)$.

Remarks. 1. The proof of this theorem will be given by studying the energy difference between a suitably chosen Roth-state and $|F\rangle$ in the thermodynamic limit, as $N /|\Lambda|$ tends to $\rho$. As it will be seen, the $\rho$-dependence of this difference 
is governed by the Van Hove singularities [9] in the free electron density of states. This may indicate why the theorem fails in one dimension and why closed-packed lattices need special care. One may conjecture that the smallest positive $\rho$ corresponding to a Van Hove singularity is a lower bound to $\rho_{0}$.

2. The result for the triangular and $f c c$ lattices could probably be improved by showing that $\rho_{0}=1$. For $\mathbb{Z}^{v}(v \geqq 2)$ and the $b c c$ lattice, however, the lowest lying Roth-state has higher energy than $|F\rangle$ in an interval $\rho_{1}<\rho<1$, and the energy difference is nonvanishing as $N$ goes to infinity.

Let us observe that for most values of $N$ the absence of ground states with $S=N / 2$ derives from the following hypothesis: For $U>0, H_{U}$ can have at most a unique ground state with a given $S$ and with $S=S^{z}$. Indeed, one can easily see that for most values of $N$ the lowest level in the subspace $S=S^{z}=N / 2$ is degenerated, so it could not be a ground state. For example, in the case of $\mathbb{L}=\mathbb{Z}^{v}$ and $t_{x y}=t$ one would obtain that any interval of length $2 v$ contains at most a single $N$ such that $S=N / 2$ can occur in a ground state.

The following theorem shows that in some cases when the lowest level in the subspace $S=S^{z}=N / 2$ is degenerated, the ground state is indeed outside this space.

Theorem 2. (i) $H_{U \leqq \infty}$ on the ring $[0, L-1]$ with $N<L$ has no highest-spin ground state if either $t>0$ and $N$ is even or $t<0$ and $L-N$ is even.

(ii) There exists $c>0$ independent of $\Lambda$ and $N$ such that for $v$-gonal lattices, if $N /|\Lambda| \geqq \rho$, then

$$
E_{\Lambda, N}(N / 2-1)-E_{\Lambda, N}(N / 2) \leqq \frac{c}{\rho} L_{\max }^{-2}
$$

(iii) $H_{U \leqq \infty}$ on $\mathbb{Z}^{v}$ with one hole $(N=|\Lambda|-1)$ has no highest-spin ground state if $t>0$ and $L_{i}$ is odd for some $i$.

(iv) $H_{U \leqq \infty}$ on any $v$-gonal lattice with two holes $(N=|\Lambda|-2)$, on the triangular lattice with $n=2,3,4$ holes and $L_{1}=L_{2}$ and on the bcc lattice with $n=2, \ldots, 6$ holes and $L_{1}=L_{2}=L_{3}(N=|\Lambda|-n)$ has no highest-spin ground state if the eigenvalue $E_{\Lambda,|\Lambda|-1}((|\Lambda|-1) / 2)$ is non-degenerated (for $|\Lambda|-1$ electrons, in the subspaces $\left.S=S^{z}=(|\Lambda|-1) / 2\right)$.

Remarks. 1. In one dimension (i) exhausts all the cases when the level $E_{\Lambda, N}(N / 2)$ is degenerated. A complementary discussion on the existence of highest-spin ground states is presented in the Appendix.

2. Equation (1.10) applies to the case of Nagaoka with a stronger bound $\sim 1 / N L_{\max }^{2}$ (cf. Eq. (3.24) and shows that the gap to the ferromagnetic ground state disappears rapidly in the thermodynamic limit. The fact that Nagaoka's result does not extend to more than one hole has long been suspected. For two recent interesting papers on the two-hole problem see Fang et al. [10] and Douçot and Wen [11].

3. The condition in (iv) is easy to check (see the proof) and implies the degeneracy of the level $E_{\Lambda, N}(N / 2)$. For example, $E_{\Lambda,|\Lambda|-1}((|\Lambda|-1) / 2)$ is nondegenerated and equals $-\Sigma_{|x|=a_{0}}\left|t_{x}\right|$ if $t_{x}<0$.

4. Theorem 2 makes use of another family of trial states, different of the Roth states. As it is shown in the proof, in the case of half-filling both families reduce to the full set of $S=N / 2-1$ eigenstates of the spin-1/2 Heisenberg Hamiltonian. In this sense, they are natural generalizations of the spin-wave excitations. 
The above theorems are valid for any $U \leqq \infty$ but in a limited domain of the electron number $N$. For the sake of completeness, one may notice that for any $N>1$ the ground state cannot have $S=N / 2$ if $U$ is small enough. Indeed, from the minimax principle [12] it follows that, e.g., for $N$ even

Therefore, if

$$
E_{\Lambda, N}(0) \leqq 2 E_{\Lambda, N / 2}(N / 4)+\frac{N}{2} U
$$

$$
U<U_{0}(\Lambda, N)=\frac{2}{N}\left[E_{\Lambda, N}(N / 2)-2 E_{\Lambda, N / 2}(N / 4)\right]
$$

then

$$
E_{\Lambda, N}(N / 2)-E_{\Lambda, N}(0)>\frac{N}{2}\left[U_{0}(\Lambda, N)-U\right] .
$$

The proof of the two theorems is given in Sect. 2 and 3. In an Appendix a proposition is proved on the continuity of the eigenvalues and eigenvectors of $H_{U}$ at $U=\infty$, and the appearance of highest-spin ground states in one dimension for $U=\infty$ is discussed.

I thank Hervé Kunz and Andreas Mielke for many illuminating discussions on the Hubbard model.

\section{Proof of Theorem 1}

The reciprocal lattice associated with a Bravais lattice $\mathbb{L}$ (Eq. 1.5) is

$$
\hat{\mathbb{L}}=\left\{n_{1} b_{1}+\ldots+n_{v} b_{v} \mid n_{i} \in \mathbb{Z}\right\},
$$

where $b_{1}, \ldots, b_{v} \in \mathbb{R}^{v}$ are defined by

$$
\left(a_{i}, b_{j}\right)=2 \pi \delta_{i j}
$$

The Brillouin zone assigned to a finite part $\Lambda$ (cf. Eq. 1.7) of $\mathbb{L}$ is the factor group

$$
\hat{\Lambda}=\left\{\frac{n_{1}}{L_{1}} b_{1}+\cdots+\frac{n_{v}}{L_{v}} b_{v} \mid n_{i} \in \mathbb{Z}\right\} / \hat{\mathbb{L}} .
$$

One defines the Fourier transform of $c_{x \sigma}$ as

$$
c_{k \sigma}=|\Lambda|^{-1 / 2} \sum_{x \in \Lambda} c_{x \sigma} e^{i(k, x)} \text { for } k \in \hat{\Lambda}
$$

Then

$$
c_{x \sigma}=|\Lambda|^{-1 / 2} \sum_{k \in \hat{\Lambda}} c_{k \sigma} e^{-i(k, x)}
$$

and the kinetic energy takes on the form

$$
H_{0}=\sum_{k \in \hat{\Lambda}} \varepsilon_{k}\left(n_{k \uparrow}+n_{k \downarrow}\right) .
$$

Here $n_{k \sigma}=c_{k \sigma}^{\dagger} c_{k \sigma}$ and

$$
\varepsilon_{k}=-\sum_{|x|=a_{0}} t_{x} e^{i(k, x)}=-\sum_{|x|=a_{0}} t_{x} \cos (k, x) .
$$


To any set $A$ of $N$ different elements of $\hat{\Lambda}$ there exists an eigenstate of $H_{U}$ in the subspace $S=S^{z}=N / 2$ :

$$
\begin{aligned}
H_{U}|A\rangle & =E_{A}|A\rangle, \\
|A\rangle & =\prod_{k \in A} c_{k \uparrow}^{\dagger}|0\rangle, \\
E_{A} & =\sum_{k \in A} \varepsilon_{k} .
\end{aligned}
$$

The vacuum state $|0\rangle$ is defined by $c_{x \sigma}|0\rangle=0$ for all $x, \sigma$.

Choose a set $F \subset \hat{\Lambda},|F|=N$ such that

$$
E_{F}=E_{\Lambda, N}(N / 2)=\min \left\{E_{A}|A \subset \hat{\Lambda},| A \mid=N\right\} .
$$

An $N$-electron Roth state with wave vector $q$ is

$$
R_{q}=|\Lambda|^{-1 / 2} \sum_{x \in \Lambda} e^{-i(q, x)} S_{x}^{-} c_{x \uparrow}^{\dagger} c_{k_{F} \uparrow}|F\rangle,
$$

where $k_{F} \in F$ is a wave vector with maximal energy:

$$
\varepsilon_{k_{F}}=\max _{k \in F} \varepsilon_{k} \equiv \varepsilon_{F} .
$$

This is a state with no doubly occupied site. One can check easily that

$$
S^{+} R_{q}=c_{q \uparrow}^{\dagger} c_{k_{F} \uparrow}|F\rangle=0
$$

if and only if $q \in F, q \neq k_{F}$ and hence $R_{q}$ has $S=S^{z}=N / 2-1$ if and only if $k_{F} \neq q \in F$.

To obtain the energy of the state $R_{q}$ we need to compute $\left\langle R_{q} \mid R_{q}\right\rangle$ and $\left\langle R_{q}\left|H_{0}\right| R_{q}\right\rangle$. The first is simple,

$$
\left\langle R_{q} \mid R_{q}\right\rangle=\frac{n+1}{|\Lambda|},
$$

where $n$ denotes the number of holes,

$$
n=|\Lambda|-N \text {. }
$$

To compute the expectation value of $H_{0}$, it is useful to write $R_{q}$ completely in $k$-space representation:

$$
R_{q}=\frac{n+1}{|\Lambda|} c_{q \downarrow}^{\dagger}\left|F_{0}\right\rangle-\frac{1}{|\Lambda|} \sum_{k \in F_{0}} \sum_{k^{\prime} \in F_{0}^{c}} c_{q+k-k^{\prime} \downarrow}^{\dagger} c_{k^{\prime} \uparrow}^{\dagger} c_{k \uparrow}\left|F_{0}\right\rangle
$$

where $F_{0}=F \backslash\left\{k_{F}\right\}$ and $F_{0}^{c}=\hat{\Lambda} \backslash F_{0}$. The state $\left|F_{0}\right\rangle$ is an eigenstate with minimal eigenvalue $\left(E_{F_{0}}\right)$ of $H_{U}$ for $N-1$ electrons and $S=S^{z}=(N-1) / 2$. With (2.15) one gets

$$
\begin{aligned}
\left\langle R_{q}\left|H_{0}\right| R_{q}\right\rangle= & \left(\frac{n+1}{|\Lambda|}\right)^{2} \varepsilon_{q}+\frac{n+1}{|\Lambda|}\left(1-\frac{1}{n+1}\right) E_{F_{0}} \\
& +|\Lambda|^{-2} \sum_{k \in F_{0}} \sum_{k^{\prime} \in F_{0}^{c}} \varepsilon_{q+k-k^{\prime}}
\end{aligned}
$$


Let us assume, at first, that the level $E_{F_{0}}$ is nondegenerated for $N-1$ electrons and $S=S^{z}=(N-1) / 2$. Then, with the choice $t_{x}=t$, the sets $F_{0}$ and $F_{0}^{c}$ are invariant under the point group transformations of $\mathbb{L}$. If $O$ is an element of the point group then

$$
\sum_{k \in F_{0}} e^{i(k, x)}=\sum_{k \in F_{0}} e^{i\left(O^{-1} k, x\right)}=\sum_{k \in F_{0}} e^{i(k, O x)}
$$

and similar equation holds with $F_{0}^{c}$. Let $|x|=a_{0}$. By the definition of the $v$-gonal lattice,

$$
\sum_{k \in F_{0}} e^{i(k, x)}=\sum_{k \in F_{0}} \sum_{|y|=a_{0}} t_{y} e^{i(k, y)} / \sum_{|y|=a_{0}} t_{y}=E_{F_{0}} / \varepsilon_{0}
$$

and

$$
\sum_{k^{\prime} \in F_{0}^{c}} e^{-i\left(k^{\prime}, x\right)}=-E_{F_{0}} / \varepsilon_{0}
$$

In (2.19) we used

$$
E_{\hat{\Lambda}}=\sum_{k \in \hat{\Lambda}} \varepsilon_{k}=0 .
$$

With the help of (2.18) and (2.19),

$$
\begin{aligned}
\sum_{k \in F_{0}} \sum_{k^{\prime} \in F_{0}^{c}} \varepsilon_{q+k-k^{\prime}} & =-\sum_{|x|=a_{0}} t_{x} e^{i(q, x)} \sum_{k \in F_{0}} e^{i(k, x)} \sum_{k^{\prime} \in F_{0}^{c}} e^{-i\left(k^{\prime}, x\right)} \\
& =-\varepsilon_{q} E_{F_{0}}^{2} / \varepsilon_{0}^{2} .
\end{aligned}
$$

Introduce

$$
\delta(\Lambda, N)=\frac{n+1}{|\Lambda|}, \quad \mu(\Lambda, N)=-\frac{E_{F_{0}}}{n+1},
$$

the hole concentration and average hole energy, respectively. Then the energy difference between $R_{q}$ and $|F\rangle$ is

$$
\lambda_{q}^{0}(\Lambda, N)=\frac{\left\langle R_{q}\left|H_{0}-E_{F}\right| R_{q}\right\rangle}{\left\langle R_{q} \mid R_{q}\right\rangle}=\mu-\varepsilon_{F}+\delta \varepsilon_{q}\left(1-\mu^{2} / \varepsilon_{0}^{2}\right) .
$$

In general, the level $E_{F_{0}}$ is degenerated and a correction has to be added to Eq. (2.23). Let $N^{\prime}$ be the smallest integer $\geqq N-1$ such that the lowest eigenvalue of $H_{U}$ for $N^{\prime} / 2$ is nondegenerated. Lengthy but straightforward calculation shows that the corrected energy difference is

$$
\begin{aligned}
& \lambda_{q}(\Lambda, N)=\lambda_{q}^{0}(\Lambda, N)+r(\Lambda, N), \\
& |r(\Lambda, N)| \leqq 8\left|\varepsilon_{0}\right| \frac{N^{\prime}-N+1}{|\Lambda|} .
\end{aligned}
$$

Now

$$
N^{\prime}-N+1 \leqq\left|\left\{k \in \hat{\Lambda} \mid \varepsilon_{k}=\varepsilon_{F}\right\}\right| \leqq \text { const } \times N^{(v-1) / v}
$$

so that $r(\Lambda, N) \rightarrow 0$ as $|\Lambda| \rightarrow \infty$. 
We want to analyze $\lambda_{q}(\Lambda, N)$ in the thermodynamic limit. Let $L_{1}, \ldots, L_{v} \rightarrow \infty$ and $N /|\Lambda| \rightarrow \rho \in[0,1]$. Then $\delta \rightarrow 1-\rho, \varepsilon_{F} \rightarrow \hat{\varepsilon}(\rho)$ and $\mu \rightarrow \hat{\mu}(\rho)$, where $\hat{\varepsilon}$ and $\hat{\mu}$ are determined via the equations

$$
\rho=\frac{v}{(2 \pi)^{v}} \int_{\varepsilon_{k}<\hat{\varepsilon}} d^{v} k
$$

and

$$
\hat{\mu}=\int_{\varepsilon_{k}>\hat{\varepsilon}} \varepsilon_{k} d^{v} k / \int_{\varepsilon_{k}>\hat{\varepsilon}} d^{v} k .
$$

The integration is carried out in $\mathbb{R}^{v} / \hat{\mathbb{L}}$ and

$$
v=\left|\operatorname{det}\left(e_{j}, a_{i}\right)\right|
$$

is the volume of the primitive cell of $\mathbb{L}$ (the $e_{j}$ are orthogonal unit vectors). The existence of the limits is a well-known fact in solid state physics. The speed of convergence depends on the way $L_{1}, \ldots, L_{v}$ tend to infinity.

Since $\mu<\left|\varepsilon_{0}\right|, \lambda_{q}$ can be minimized by choosing $q$ such that it makes $\varepsilon_{q}$ minimal. The value

$$
\varepsilon_{\min }=\min \left\{\varepsilon_{k} \mid k \in \mathbb{R}^{v}\right\}
$$

can be attained at least in the thermodynamic limit. The resulting limit for $\lambda_{q}(\Lambda, N)$ is

$$
\lambda(\rho)=\hat{\mu}-\hat{\varepsilon}+(1-\rho) \varepsilon_{\min }\left(1-\hat{\mu}^{2} / \varepsilon_{0}^{2}\right) .
$$

In one dimension $\varepsilon_{\min }=-2|t|, \varepsilon_{0}=-2 t$ and

$$
\hat{\varepsilon}(\rho)=-2|t| \cos \pi \rho, \quad \hat{\mu}(\rho)=\frac{2|t| \sin \pi(1-\rho)}{\pi(1-\rho)} .
$$

One can check that $\lambda(\rho)>0$ for $0<\rho<1 ; \lambda^{\prime}(0)=4|t|$ and $\lambda^{\prime}(1)=0$.

In general, $\lambda(\rho)$ is continuous and

$$
\lambda(0)=\lambda(1)=0
$$

because

$$
\hat{\varepsilon}(0)=\varepsilon_{\min }, \quad \hat{\mu}(0)=0 \quad \text { and } \quad \hat{\varepsilon}(1)=\hat{\mu}(1)=\varepsilon_{\max }=\max _{k} \varepsilon_{k} .
$$

Furthermore, $\lambda(\rho)$ is continuously differentiable for $0<\rho<1$. To see this, notice that

$$
\hat{\mu}^{\prime}(\rho)=[\hat{\mu}(\rho)-\hat{\varepsilon}(\rho)] /(1-\rho) .
$$

The continuity of $\hat{\varepsilon}^{\prime}(\rho)$ can be seen by looking at

$$
\frac{d \rho}{d \hat{\varepsilon}}=\frac{v}{(2 \pi)^{v}} \int_{\varepsilon_{k}=\hat{\varepsilon}} \frac{d S}{\left|\operatorname{grad} \varepsilon_{k}\right|} .
$$

This is positive nonvanishing in $\left(\varepsilon_{\min }, \varepsilon_{\max }\right)$ and hence $\hat{\varepsilon}^{\prime}(\rho) \geqq 0$ finite in $\rho \in(0,1)$. The zeros of grade $\varepsilon_{k}$ give rise to Van Hove singularities in $\rho(\hat{\varepsilon})$ at $\varepsilon_{\min }, \varepsilon_{\max }$ and at the saddle points of $\varepsilon_{k}$ [9]. Since the limit of $d \rho / d \hat{\varepsilon}$ in these singularities exists (may be $+\infty$ ), the limit of $\hat{\varepsilon}^{\prime}(\rho)$ also exists (may be $+\infty$ at $\rho=0$ or 1 ). 
We want to show that $\lambda(\rho)<0$ near $\rho=0$ by proving that $\lambda^{\prime}(\rho)$ has a negative limit as $\rho$ tends to 0. From Eqs. (2.30), (2.33) and (2.34) one obtains

$$
\begin{aligned}
\lim _{\rho \rightarrow 0} \lambda^{\prime}(\rho) & =-2 \varepsilon_{\min }-\lim _{\rho \rightarrow 0} \hat{\varepsilon}^{\prime}(\rho) \\
\lim _{\rho \rightarrow 1} \lambda^{\prime}(\rho) & =\frac{1}{2} \lim _{\rho \rightarrow 1}\left[\frac{\lambda(\rho)-\lambda(1)}{\rho-1}+\lambda^{\prime}(\rho)\right] \\
= & -\varepsilon_{\min }\left[1-\frac{\varepsilon_{\max }^{2}}{\varepsilon_{0}^{2}}\right]-\frac{1}{2} \lim _{\rho \rightarrow 1} \hat{\varepsilon}^{\prime}(\rho) .
\end{aligned}
$$

The extremal values of $\rho$ correspond to the extremal values of

$$
f(k)=-\varepsilon_{k} / t=\sum_{|x|=a_{0}} \cos (k, x) .
$$

The maximum of $f(k)$ is attained in a unique point of $\mathbb{R}^{v} / \hat{\mathbb{L}}, k=0$. The minimum may be reached in a unique point, in a finite number of different points or on a continuous set, depending on the lattice type. The different cases are discussed separately.

(i) $\mathbb{Z}^{v}$ for $v \geqq 3$ and the bcc lattice

The maximum of $f(k)$ is obtained also in a unique point, $k=\frac{1}{2}\left(b_{1}+\ldots+b_{v}\right)$. Consider the limit $\rho \rightarrow \rho^{*}=0$ or 1 and let $k^{*}=0$ or $\left(b_{1}+\ldots+b_{v}\right) / 2$ be the corresponding extremum of $f(k)$. For $\rho$ near to $\rho^{*}$, on the surface $\varepsilon_{k}=\hat{\varepsilon}(\rho)$ about $k^{*}$ we have $\left|\operatorname{grad} \varepsilon_{k}\right| \sim\left|k-k^{*}\right|$; if $r$ is the average value of $\left|k-k^{*}\right|$ on the surface then $d \rho / d \hat{\varepsilon}=O\left(r^{v-2}\right)$. This is valid also in one dimension, thus

$$
\lim _{\rho \rightarrow \rho^{*}} \hat{\varepsilon}^{\prime}(\rho)=\left\{\begin{array}{lll}
0 & \text { if } & v=1 \\
+\infty & \text { if } & v \geqq 3
\end{array} .\right.
$$

From (2.36) and (2.37), in one dimension

$$
\lambda^{\prime}(0)=-2 \varepsilon_{\min }=4|t|, \quad \lambda^{\prime}(1)=0
$$

in agreement with what we obtained from Eq. (2.31). In the case of $v \geqq 3$ and of the $b c c$ lattice, $\lambda^{\prime}(\rho)$ tends to $-\infty$ if $\rho$ goes to 0 or 1 . This and $\lambda(0)=\lambda(1)=0$ imply the existence of $\rho_{0}, \rho_{1}, 0<\rho_{0} \leqq \rho_{1}<1$, such that $\lambda(\rho)<0$ if $0<\rho<\rho_{0}$ and $\lambda(\rho)>0$ if $\rho_{1}<\rho<1$.

(ii) Square lattice

For $v=2, \hat{\varepsilon}^{\prime}(\rho)=O(1)$ as $\rho \rightarrow \rho^{*}$ and, hence, more careful considerations are necessary. For the square lattice $f(k)$ has a unique maximum and minimum. Let $k^{*}$ be the extremum corresponding to $\rho^{*}, e$ any unit vector and $s e+k^{*}$ with $s>0$ the single vector solving

$$
\hat{\varepsilon}(\rho)=\varepsilon_{s e+k^{*}}
$$

This makes $s$ a differentiable function of $\rho$. Now

$$
\hat{\varepsilon}^{\prime}\left(\rho^{*}\right)=\left(\frac{d \varepsilon_{s e+k^{*}}}{d s^{2}}\right)_{s=0}\left(\frac{d s^{2}}{d \rho}\right)_{\rho=\rho^{*}} .
$$


One then finds

$$
\left(\frac{d \varepsilon_{s e+k^{*}}}{d s^{2}}\right)_{s=0}=\frac{t}{2} \sum_{|x|=a_{0}}(e, x)^{2} \cos \left(k^{*}, x\right) .
$$

Notice that this is independent of $e$. Using that $\varepsilon_{k}$ has a quadratic extremum in $k^{*}$, Eq. (2.26) yields

$$
\left|\rho-\rho^{*}\right|=\frac{v}{(2 \pi)^{2}} \pi s^{2}+O\left(s^{4}\right) .
$$

By inverting this relation,

$$
\left|\frac{d s^{2}}{d \rho}\right|_{\rho=\rho^{*}}=\frac{4 \pi}{v}
$$

which, together with (2.42) and (2.43) gives

$$
\hat{\varepsilon}^{\prime}\left(\rho^{*}\right)=\frac{2 \pi|t|}{v}\left|\sum_{|x|=a_{0}}(e, x)^{2} \cos \left(k^{*}, x\right)\right| .
$$

Using $v=a_{0}^{2}$ finally we get $\hat{\varepsilon}^{\prime}(0)=\hat{\varepsilon}^{\prime}(1)=4 \pi|t|$ and

$$
\begin{aligned}
& \lambda^{\prime}(0)=-4(\pi-2)|t|, \\
& \lambda^{\prime}(1)=-2 \pi|t| .
\end{aligned}
$$

With Eq. (2.32) this shows that $\lambda(\rho)$ is negative near $\rho=0$ and positive near $\rho=1$.

(iii) Triangular lattice

We distinguish between two cases.

(1) $\rho^{*}$ corresponds to the maximum of $f(k)$, i.e., $k^{*}=0$. Then Eqs. $(2.41-46)$ remain valid. With

one obtains

$$
v=a_{0}^{2} \sqrt{3} / 2, \quad \sum_{|x|=a_{0}}(e, x)^{2}=3 a_{0}^{2}
$$

$$
\hat{\varepsilon}^{\prime}\left(\rho^{*}\right)=4 \pi \sqrt{3}|t|=\left\{\begin{array}{ll}
\hat{\varepsilon}^{\prime}(0) & \text { if } t>0 \\
\hat{\varepsilon}^{\prime}(1) & \text { if } t<0
\end{array} .\right.
$$

(2) $\rho^{*}$ corresponds to the minimum of $f(k)$. This is attained in two nonequivalent points, e.g., in

$$
k_{1}=\left(b_{1}+b_{2}\right) / 3, \quad k_{2}=\left(-b_{1}+2 b_{2}\right) / 3
$$

at the value $f\left(k_{i}\right)=-3$. The two points are related by symmetry, therefore their contributions in Eq. (2.35) are equal. We choose two unit vectors $e_{1}$ and $e_{2}$ such that if $k_{2}=O k_{1}$ for a point group element $O$ then $e_{2}=O e_{1}$. Thus,

$$
\hat{\varepsilon}=\varepsilon_{s e_{1}+k_{1}}=\varepsilon_{s e_{2}+k_{2}}
$$

for the same $s>0$. Equations (2.42) and (2.43) remain valid if $e$ and $k^{*}$ are replaced by $e_{i}$ and $k_{i}$. Equation (2.44) is changed to

$$
\left|\rho-\rho^{*}\right|=2 \frac{v}{(2 \pi)^{2}} \pi s^{2}+O\left(s^{3}\right),
$$


so that

$$
\left|\frac{d s^{2}}{d \rho}\right|_{\rho=\rho^{*}}=\frac{2 \pi}{v} .
$$

With $\cos \left(k_{i}, x\right)=-1 / 2$ if $|x|=a_{0}$, we find

where (2.48) was used.

$$
\hat{\varepsilon}^{\prime}\left(\rho^{*}\right)=\sqrt{3} \pi|t|= \begin{cases}\hat{\varepsilon}^{\prime}(0) & \text { if } t<0 \\ \hat{\varepsilon}^{\prime}(1) & \text { if } t>0\end{cases}
$$

Noticing that

$$
\begin{aligned}
& \varepsilon_{\max }(t<0)=-\varepsilon_{\min }(t>0)=6|t| \\
& \varepsilon_{\max }(t>0)=-\varepsilon_{\min }(t<0)=3|t|
\end{aligned}
$$

one may summarize as follows:

$$
\lambda^{\prime}(0)= \begin{cases}-4 \sqrt{3}(\pi-\sqrt{3}) t & \text { if } t>0 \\ (6-\sqrt{3} \pi)|t| & \text { if } t<0\end{cases}
$$

and

$$
\lambda^{\prime}(1)=\left\{\begin{array}{lll}
\frac{1}{2}(9-\sqrt{3} \pi) t & \text { if } t>0 \\
-2 \sqrt{3} \pi|t| & \text { if } t<0
\end{array} .\right.
$$

Equations (2.56) and (2.57) together with (2.32) show that for $t>0, \lambda(\rho)$ is negative (and for $t<0$ it is positive) near $\rho=0$ and $\rho=1$.

(iv) The fcc lattice

If $t>0$ and $\rho \rightarrow 0$ then $\hat{\varepsilon}(\rho) \rightarrow \varepsilon_{\min }=-t f(0)=-12 t$, the minimal energy is attained in the unique point $k=0$. The discussion of (i) is valid: $\hat{\varepsilon}^{\prime}(0)=+\infty$ and $\lambda^{\prime}(0)=-\infty$, so that $\lambda(\rho)$ is negative near $\rho=0$. Similarly, if $t<0$ then $\lambda^{\prime}(1)=-\infty$, hence $\lambda(\rho)>0$ near $\rho=1$.

If $t>0$, as $\rho \rightarrow 1$ the energy $\hat{\varepsilon}(\rho)$ tends to $\varepsilon_{\max }^{3}=-t \min f(k)=4 t$. Now $f(k)$ is minimal on a continuous set which is the union of the line segments

$$
k_{i j}^{*}(\beta)=\frac{1}{2}\left(b_{j}+b_{k}\right)+\beta\left(b_{k}+b_{i}\right), \quad-\frac{1}{4} \leqq \beta \leqq \frac{1}{4} .
$$

Here, $i, j, k \in\{1,2,3\}$ are different. The reciprocal of the $f c c$ lattice is the bcc lattice. The first Brillouin zone (the Voronoy polyhedron of $\hat{\mathbb{L}}$, a particular representation of $\mathbb{R}^{v} / \hat{\mathbb{L}}$ ) is a truncated octahedron with 8 hexagonal and 6 square faces. The segments (2.58) are the diagonals of the square faces. The limit of $d \rho / d \varepsilon$ as $\varepsilon$ goes to $4 t$ can be computed in the following way. Fix an $f_{0}>-4, f_{0}+4 \ll 1$, and consider the surface $f(k)=f_{0}$. The plane passing through $k_{i j}^{*}(\beta)$ perpendicular to $b_{k}+b_{i}$ cuts this surface in a nearly ellipse. If $k(\beta, \varphi)$ denotes the point of this curve at an angle $\varphi$ to the vector $b_{j}+b_{k}$ then

$$
\begin{aligned}
\mid \operatorname{grad} f(\beta, \varphi)) \mid= & \frac{1}{2} a^{2} r(\varphi)\left[(1+\cos \alpha)^{2} \cos ^{2} \varphi+(1-\cos \alpha)^{2} \sin ^{2} \varphi\right]^{1 / 2} \\
& +o\left(f_{0}+4\right),
\end{aligned}
$$

where

$$
r(\varphi)=\left|k(\beta, \varphi)-k_{i j}^{*}(\beta)\right|, \quad \alpha=2 \pi \beta
$$


A. Sütő

and $a$ is the lattice constant of the conventional cubic cell of the $f c c$ lattice. With (2.59) we obtain that

$$
\begin{aligned}
|t|(d \rho / d \varepsilon)_{\varepsilon=4 t} & =\lim _{f_{0} \rightarrow-4} \frac{v}{(2 \pi)^{3}} \int_{f(k)=f_{0}} \frac{d S}{|\operatorname{grad} f(k)|} \\
& =\frac{6}{\pi^{3}} \int_{0}^{\pi / 2} \int_{0}^{\pi / 2} \frac{d \alpha d \varphi}{\left[(1+\cos \alpha)^{2}-4 \cos \alpha \sin ^{2} \varphi\right]^{1 / 2}} \\
& >\frac{6}{\pi^{3}} \mathbf{K}(0)>0.3 .
\end{aligned}
$$

In the above equation $\mathbf{K}$ is the complete elliptic integral of the first kind.

Now from Eqs. (2.37) and (2.61) for $t>0$ one finds

$$
\lambda^{\prime}(1)=\frac{32}{3} t-\frac{1}{2}\left[(d \rho / d \varepsilon)_{\varepsilon=4 t}\right]^{-1}>9 t
$$

which means that $\lambda(\rho)<0$ near $\rho=1$. On the other hand, if $t<0$ then

$$
\lambda^{\prime}(0)=8|t|-1 /(d \rho / d \varepsilon)_{\varepsilon=4 t}>\frac{14}{3}|t|,
$$

so that $\lambda(\rho)$ is positive near $\rho=0$. In summary, for $t>0, \lambda(\rho)$ is negative (and for $t<0$ it is positive) near $\rho=0$ and $\rho=1$.

To finish the proof of the theorem, we have to compare the thermodynamic limit (2.30) with the finite-volume expression (2.24). It suffices to notice that if

$$
\rho \leqq N /|\Lambda| \leqq \rho_{0}-\rho
$$

for some $\rho>0$ then

$$
\left|\lambda_{q}(\Lambda, N)-\lambda(N /|\Lambda|)\right|<|\lambda(N /|\Lambda|)|
$$

will hold true whenever $L_{\min }$ is sufficiently large. Similar argument proves the special result for the triangular and $f c c$ lattices.

\section{Proof of Theorem 2}

For a nonzero $q \in \hat{\Lambda}$, let

$$
\Sigma_{q}^{z}=\left(\sum_{x \in \Lambda} S_{x}^{z} e^{i(q, x)}\right) S^{-}
$$

Given a set $F \subset \hat{\Lambda},|F|=N$, we define a family of trial states by

$$
\psi_{q}=\left(N-S^{-} S^{+}\right) \Sigma_{q}^{z}|F\rangle \text {. }
$$

For $|F\rangle$ the definition (2.9) applies. The effect of $\Sigma_{q}^{z}$ on $|F\rangle$ is to introduce a component with $S=N / 2-1$ without creating doubly occupied sites. Then $N-S^{-} S^{+}$singles out this component. The result is

$$
\begin{aligned}
\psi_{q}= & -N \sum_{k \in F, k-q \in F} c_{k-q \downarrow}^{\dagger} c_{k \uparrow}|F\rangle-(N-1) \sum_{k \in F, k-q \notin F} c_{k-q \downarrow}^{\dagger} c_{k \uparrow}|F\rangle \\
& -\sum_{k \in F, k-q \notin F \boldsymbol{F} k^{\prime} \in F \backslash\{k\}} c_{k-q \uparrow}^{\dagger} c_{k^{\prime} \downarrow}^{\dagger} c_{k \uparrow} c_{k^{\prime} \uparrow}|F\rangle .
\end{aligned}
$$


We make here a detour to discuss the particular case of half-filling. If $N=|\Lambda|$ then the only possible choice for $F$ is $F=\hat{\Lambda}$, and $\psi_{q}$ reduces to the first sum. One can check from Eq. (2.15) that at the same time $R_{q}$ is proportional to $\psi_{q^{\prime}}$ where $q^{\prime}=k_{F}-q$.

Proposition 1. Let $P$ be the projection to the subspace of states without doubly occupied sites (cf. Eq. A.1). Let $f(x)$ be any real valued continuous function. Fix $N=|\Lambda|$. Then $\left\{\psi_{q} \mid q \in \hat{\Lambda}, q \neq 0\right\}$ is a complete set of eigenstates of $P f\left(H_{0}\right) P$ in the subspace $S=S^{z}=N / 2-1$, and

$$
P f\left(H_{0}\right) P \psi_{q}=\sum_{k \in \hat{\Lambda}} f\left(\varepsilon_{k-q}-\varepsilon_{k}\right) \psi_{q} .
$$

Proof.

$$
\psi_{q}=\sum_{k \in \hat{\Lambda}} c_{k-q \downarrow}^{\dagger} c_{k \uparrow}|\hat{\Lambda}\rangle
$$

for $q \in \hat{\Lambda}, q \neq 0$ form an orthogonal basis in the subspace of states with $S=S^{z}=N / 2-1$ and without doubly occupied sites: $\left\langle\psi_{q} \mid \psi_{q^{\prime}}\right\rangle=0$ obviously if $q \neq q^{\prime}$, and the dimension of this subspace is $|\Lambda|-1$. Now

$$
H_{0} c_{k-q \downarrow}^{\dagger} c_{k \uparrow}|\hat{\Lambda}\rangle=\left(\varepsilon_{k-q}-\varepsilon_{k}\right) c_{k-q \downarrow}^{\dagger} c_{k \uparrow}|\hat{\Lambda}\rangle,
$$

therefore

$$
\begin{aligned}
\left\langle\psi_{q^{\prime}}\left|P f\left(H_{0}\right) P\right| \psi_{q}\right\rangle & =\left\langle\psi_{q^{\prime}}\left|f\left(H_{0}\right)\right| \psi_{q}\right\rangle \\
& =\sum_{k, k^{\prime} \in \hat{\Lambda}}\left\langle\hat{\Lambda}\left|c_{k^{\prime} \uparrow}^{\dagger} c_{k^{\prime}-q^{\prime} \downarrow} f\left(\varepsilon_{k-q}-\varepsilon_{k}\right) c_{k-q \downarrow}^{\dagger} c_{k \uparrow}\right| \hat{\Lambda}\right\rangle \\
& =\delta_{q q^{\prime}} \sum_{k \in \hat{\Lambda}} f\left(\varepsilon_{k-q}-\varepsilon_{k}\right)
\end{aligned}
$$

which proves the assertion.

At half-filling, $P f\left(H_{0}\right) P$ can be expressed in terms of spin operators. For example, one can easily check that

$$
P H_{0}^{2} P=-4 t^{2} \sum_{|x-y|=a_{0}}\left[S_{x}^{z} S_{y}^{z}+\frac{1}{2}\left(S_{x}^{+} S_{y}^{+}+S_{x}^{-} S_{y}^{-}\right)-\frac{1}{4}\right],
$$

the ferromagnetic Heisenberg Hamiltonian.

Let us return to Eq. (3.3). One finds after some computation that

$$
\left\langle\psi_{q} \mid \psi_{q}\right\rangle=N^{3}-N|F \backslash(F+q)|,
$$

where $F+q=\{k+q \mid k \in F\}$, and

$$
\left\langle\psi_{q}\left|H_{0}-E_{F}\right| \psi_{q}\right\rangle=N^{2} \sum_{k \in F}\left(\varepsilon_{k-q}-\varepsilon_{k}\right)-N \sum_{k \in F, k-q \notin F}\left(\varepsilon_{k-q}-\varepsilon_{k}\right) .
$$

The energy difference between the states $\psi_{q}$ and $|F\rangle$ is

$$
\lambda_{q}=Z^{-1}\left\{E_{F-q}-E_{F}-N^{-1} \sum_{k \in F, k-q \neq F}\left(\varepsilon_{k-q}-\varepsilon_{k}\right)\right\},
$$


where

$$
Z=N-N^{-1}|F \backslash(F+q)|
$$

Let $1<N<|\Lambda|$ and fix $F$ as in Eq. (2.9). If $|F\rangle$ is the unique state minimizing $H_{0}$ in the subspace $S=S^{z}=N / 2$, then $\lambda_{q}>0$. This can be seen by rewriting $\lambda_{q}$ as

$$
\lambda_{q}=Z^{-1}\left\{\sum_{k \in F, k-q \in F}\left(\varepsilon_{k-q}-\varepsilon_{k}\right)+\left(1-N^{-1}\right) \sum_{k \in F, k-q \notin F}\left(\varepsilon_{k-q}-\varepsilon_{k}\right)\right\} .
$$

If $F$ satisfies (2.9) then $-F=\{-k \mid k \in F\}$ does so as well. Uniqueness therefore means $-F=F$. This and $\varepsilon_{k}=\varepsilon_{-k}$ imply

$$
\sum_{k \in F, k-q \in F}\left(\varepsilon_{k-q}-\varepsilon_{k}\right)=0
$$

while each term of the second sum in (3.13) is $\geqq 0$ and, in fact, strictly positive, due to the uniqueness of $F$.

Therefore, we can expect that $\lambda_{q}<0$ only if the level $E_{\Lambda, N}(N / 2)$ is degenerated, i.e., the choice of $F$ is not unique. Consider now the cases enumerated in the theorem.

(i) In one dimension, if $t>0$ then $\varepsilon_{\min }=\varepsilon_{0}$. For $N=2 m+1$, the unique set $F$ satisfying $E_{F}=E_{\Lambda, N}(N / 2)$ is

$$
F=\left\{0, \pm \frac{2 \pi}{L}, \ldots, \pm \frac{2 \pi}{L} m\right\}
$$

For $N=2 m$ there are two such sets,

$$
\begin{aligned}
& F_{1}=\left\{0, \pm \frac{2 \pi}{L}, \ldots, \pm \frac{2 \pi}{L}(m-1), \frac{2 \pi}{L} m\right\} \\
& F_{2}=\left\{0, \pm \frac{2 \pi}{L}, \ldots, \pm \frac{2 \pi}{L}(m-1),-\frac{2 \pi}{L} m\right\}=F_{1}-\frac{2 \pi}{L}
\end{aligned}
$$

Choose, for example, $F=F_{1}$ and $q=2 \pi / L$. Then

$$
E_{F-q}=E_{F}
$$

while

$$
\varepsilon_{k-q}-\varepsilon_{k}>0 \text { for } k=-\frac{2 \pi}{L}(m-1) .
$$

From Eq.(3.11), $\lambda_{q}<0$.

If $t<0$ then $\varepsilon_{\max }=\varepsilon_{0}$ and the argument applies if $N$ is replaced by $L-N$.

(ii) Obviously,

$$
E_{\Lambda, N}(N / 2-1)-E_{\Lambda, N}(N / 2) \leqq \lambda_{q} \leqq(N-1)^{-1}\left(E_{F-q}-E_{F}\right)
$$

for any nonvanishing $q$. Let $q=b_{i} / L_{i}$,

where

$$
E_{F-q}-E_{F}=E_{(F-q) \backslash F}-E_{F \backslash(F-q)},
$$

$$
|(F-q) \backslash F|=|F \backslash(F-q)|=K \leqq \min \left\{N,|\Lambda|-N, m_{0}|\Lambda| / L_{i}\right\} .
$$


Here $m_{0}=\max _{i, \varepsilon} m_{i}(\varepsilon)$, where $m_{i}(\varepsilon)$ is the maximal number of sign changes of $\varepsilon_{k}-\varepsilon$ when $k$ varies along different lines parallel to $b_{i}$. On the other hand, if $k$ is in the symmetric difference of $F$ and $F-q$ then

$$
\left|\varepsilon_{k}-\varepsilon_{F}\right| \leqq z|t| 2 \pi / L_{i},
$$

where $z$ is the coordination number on $\mathbb{L}$. Therefore

$$
\begin{aligned}
E_{F-q}-E_{F} & \leqq K\left(\varepsilon_{F}+2 \pi z|t| / L_{i}\right)-K\left(\varepsilon_{F}-2 \pi z|t| / L_{i}\right) \\
& \leqq\left(4 \pi z|t| / L_{i}\right) \min \left\{N,|\Lambda|-N, m_{0}|\Lambda| / L_{i}\right\} .
\end{aligned}
$$

We can choose $i$ such that $L_{i}=L_{\max }$. Then from Eqs. (3.19) and (3.22),

$$
\begin{aligned}
& E_{\Lambda, N}(N / 2-1)-E_{\Lambda, N}(N / 2) \\
& \quad \leqq \frac{4 \pi z|t|}{L_{\max }} \frac{N}{N-1} \min \left\{1, \frac{|\Lambda|-N}{N}, \frac{m_{0}}{L_{\max } N /|\Lambda|}\right\} .
\end{aligned}
$$

In the case considered by Nagaoka $(N=|\Lambda|-1, F$ unique) the energy difference (3.20) is proportional to $|t| L_{i}^{-2}$, so we have the better estimate

$$
E_{\Lambda, N}(N / 2-1)-E_{\Lambda, N}(N / 2) \leqq \text { const } \times|t| /\left(N L_{\max }^{2}\right)
$$

which shows that the gap to the ground state disappears extremely fast.

(iii) If $t>0$, the single hole is placed in the minimum of $f(k)$ of Eq. (2.38), which is now degenerated. The vectors minimizing $f(k)$ are

$$
\frac{1}{2} \sum_{j=1}^{v} b_{j}+\frac{1}{2} \sum_{L_{i} \text { odd }} \delta_{i} b_{i} / L_{i}, \quad \delta_{i}= \pm 1 .
$$

Let $k_{1}$ and $k_{2}=k_{1}-b_{i} / L_{i}$ be two minima. Choose $F=\hat{\Lambda} \backslash\left\{k_{1}\right\}$ and $q=b_{i} / L_{i}$, then $F-q=\hat{\Lambda} \backslash\left\{k_{2}\right\}$ and thus $E_{F-q}=E_{F}$. On the other hand, the single $k \in F$ for which $k-q \notin F$ is $k=k_{1}+b_{i} / L_{i}$, and

$$
\varepsilon_{k-q}=\varepsilon_{k_{1}}>\varepsilon_{k}=\varepsilon_{k_{1}+b_{i} / L_{i}}
$$

so that in (3.11), $\lambda_{q}<0$.

(iv) The absence of degeneracy of the level $E_{\Lambda,|\Lambda|-1}((|\Lambda|-1) / 2)$ means that $\varepsilon_{\max }=\varepsilon_{k}$ holds for a single $k=k^{*}$. This occurs if $t<0$ for any $v$-gonal lattice, when $k^{*}=0$. It occurs also if $t>0$ and all the $L_{i}$ are even for $\mathbb{Z}^{v}$ and for the bec lattice (but not for the triangular or the $f c c$ lattice), when $k^{*}=\left(b_{1}+\cdots+b_{v}\right) / 2$.

These are the cases in which Nagaoka's theorem is valid if there is a single hole. If $N=|\Lambda|-2$ then

$$
E_{\Lambda, N}(N / 2)=-\varepsilon_{k^{*}}-\varepsilon_{k_{1}},
$$

where $k_{1}$ is any of the nearest neighbors of $k^{*}$ in $\hat{\Lambda}$. If $k_{1}$ is a nearest neighbor then $k_{2}=k^{*}-\left(k_{1}-k^{*}\right)$ is also a nearest neighbor and $\varepsilon_{k_{2}}=\varepsilon_{k_{1}}$. The points $k^{*}$, $k_{1}$ and $k_{2}$ are on a line, hence the proof given in one dimension can be repeated.

$\hat{\Lambda}$ for the triangular lattice is a triangular net with lattice constant $b / L$ $\left(b=\left|b_{1}\right|=\left|b_{2}\right|\right.$ and $\left.L=L_{1}=L_{2}\right)$. One may fix $b_{1}$ and $b_{2}$ such that $\left(b_{1}, b_{2}\right)=b^{2} / 2$. For $t<0$ and $N=|\Lambda|-4$, choose for example

$$
F^{c}=\left\{k^{*}, k_{1}, k_{2}, k_{3}\right\}=\left\{0, b_{1} / L, b_{2} / L,\left(b_{1}-b_{2}\right) / L\right\}
$$


and $q=b_{1} / L$. Then $F-q=-F$ and $E_{F-q}-E_{F}=0$, while the sum in Eq. (3.11) has three positive terms for $k=k_{i}+b_{1} / L$. Hence, $\lambda_{q}<0$. For $N=|\Lambda|-3$ we may use $F^{c}=\left\{0, k_{1}, k_{2}\right\}$ and $q=b_{1} / L$.

$\hat{\Lambda}$ for the $b c c$ lattice is an $f c c$ lattice with lattice constant $b / L\left(b=\left|b_{i}\right|, L=L_{i}\right.$, $i=1,2,3)$. One can fix $b_{1}, b_{2}$ and $b_{3}$ so that they are at angles of $60^{\circ}$ to one another. In the case of 6 holes $(N=|\Lambda|-6)$ choose, for example $F$ such that

$$
\begin{aligned}
F^{c} & =\left\{k^{*}, k^{*}+k_{1}, \ldots, k^{*}+k_{5}\right\} \\
& =k^{*}+\left\{0, b_{1} / L, b_{2} / L, b_{3} / L,\left(b_{3}-b_{1}\right) / L,\left(b_{3}-b_{2}\right) / L\right\}
\end{aligned}
$$

and $q=b_{3} / L$. Then

$$
F^{c}-q=\left\{k^{*}, k^{*}-k_{1}, \ldots, k^{*}-k_{5}\right\} .
$$

Since $\varepsilon_{k^{*}+k_{i}}=\varepsilon_{k^{*}-k_{i}}$, we find again $E_{F-q}=E_{F}$. On the other hand, the sum in Eq. (3.11) has five positive terms for $k=k^{*}+k_{i}+b_{3} / L$, so we conclude that $\lambda_{q}<0$. If the number of holes is less, we can use the same $q$ and any subset of $F^{c}$ of Eq. (3.28) containing $k^{*}$. This concludes the proof of Theorem 2.

\section{A. Appendix. The $U=\infty$ Limit}

\section{A.1. Continuity of the Spectrum and the Eigenstates}

Denote $\mathscr{F}^{\Lambda}$ the $\left(4^{|\Lambda|}\right.$ dimensional) Fock space of the fermionic states on $\Lambda$. Let $P$ be the projection onto $\operatorname{ker} H_{I}$, the subspace with no doubly occupied site:

$$
P=\prod_{x \in \Lambda}\left(1-n_{x \uparrow} n_{x \downarrow}\right)
$$

Define $H_{\infty}$ by restricting $P H_{0} P$ to $\operatorname{ker} H_{I}$. If $\psi \in \operatorname{ker} H_{I}$ then

$$
\left\langle\psi\left|H_{\infty}\right| \psi\right\rangle=\left\langle\psi\left|H_{0}\right| \psi\right\rangle=\left\langle\psi\left|H_{U}\right| \psi\right\rangle \text {. }
$$

Consider a common invariant subspace $\mathscr{F}_{0} \subset \mathscr{F}^{\Lambda}$ of $H_{0}$ and $H_{I}$ (e.g., $\mathscr{F}_{0}=\mathscr{F}^{\Lambda}$ or $N$ is fixed or $N$ and $S^{z}$ are fixed, etc.). $\mathscr{F}_{0}$ will be an invariant subspace of $H_{U}$ for all $U \leqq \infty$. Let

$$
E_{1}(U) \leqq E_{2}(U) \leqq \ldots \leqq E_{K_{0}}(U)
$$

be the repeated eigenvalues in ascending order and

$$
\psi_{1}(U), \ldots, \psi_{K_{0}}(U)
$$

a set of corresponding orthonormal eigenvectors of $H_{U}$ in $\mathscr{F}_{0}$. Denote $K$ the dimension of the subspace $\mathscr{F}_{0} \cap \operatorname{ker} H_{I}$, and

$$
E_{1}(\infty) \leqq \ldots \leqq E_{K}(\infty) \text { and } \psi_{1}(\infty), \ldots, \psi_{K}(\infty)
$$

the eigenvalues and orthonormal eigenvectors of $H_{\infty}$ in $\mathscr{F}_{0}$, respectively.

Proposition 2. For $n \leqq K, E_{n}(U)$ is continuous at $U=\infty$, i.e.,

$$
\lim _{U \rightarrow \infty} E_{n}(U)=E_{n}(\infty) \text {. }
$$


Furthermore, any limit point of $\psi_{n}(U)$ as $U \rightarrow \infty$ is an eigenvector of $H_{\infty}$ with eigenvalue $E_{n}(\infty)$, and an orthonormal set of eigenvectors of $H_{\infty}$ can be obtained as such a limit.

Proof. Since $H_{I}$ is positive semidefinite, the minimax principle implies that for each $n$ the eigenvalue $E_{n}(U)$ is a monotonically increasing (and continuous) function of $U$. Let

$$
\bar{E}_{n}=\lim _{U \rightarrow \infty} E_{n}(U)
$$

where $\bar{E}_{n}$ may be infinite. In fact, we have

$$
\bar{E}_{1} \leqq \cdots \leqq \bar{E}_{K}<\infty=\bar{E}_{K+1}=\cdots=\bar{E}_{K_{0}} .
$$

This, again, follows from the minimax principle: If $n \leqq K$ then any subspace of $\mathscr{F}_{0}$ of codimension $n-1$ contains some state $\psi \neq 0$ which is in $\operatorname{ker} H_{I}$. Therefore

$$
E_{n}(U) \leqq 2 \sum\left|t_{x y}\right| \text { if } n \leqq K .
$$

The same argument shows that

$$
E_{n}(U) \geqq U-2 \sum\left|t_{x y}\right| \text { if } n>K .
$$

By a well-known consequence of the minimax principle (Theorem 6.46 in Kato [12]),

$$
\bar{E}_{n} \leqq E_{n}(\infty), \quad n=1, \ldots, K .
$$

We need the opposite inequality.

Let $\left\{U_{i}\right\}$ be an increasing sequence tending to infinity such that the eigenstates $\psi_{n}\left(U_{i}\right)$ converge simultaneously for $n=1, \ldots, K$ to some states $\psi_{n}$,

$$
\lim _{i \rightarrow \infty}\left\|\psi_{n}\left(U_{i}\right)-\psi_{n}\right\|=0, \quad n=1, \ldots, K
$$

The states $\left\{\psi_{n}\right\}_{1}^{K}$ form an orthonormal set and they are in $\mathscr{F}_{0} \cap \operatorname{ker} H_{I}$. Indeed, if $\psi_{n} \notin \operatorname{ker} H_{I}$ then

$$
\left\langle\psi_{n}\left|H_{I}\right| \psi_{n}\right\rangle>c>0,
$$

thus by (A.9),

$$
\left\langle\psi_{n}\left(U_{i}\right)\left|H_{I}\right| \psi_{n}\left(U_{i}\right)\right\rangle>c / 2
$$

for sufficiently large $i$, which implies $E_{n}\left(U_{i}\right)=O\left(U_{i}\right)$ in contradiction with (A.6). At first we show that

$$
\begin{aligned}
& E_{1}(\infty)=\bar{E}_{1} \text { and } H_{\infty} \psi_{1}=\bar{E}_{1} \psi_{1}: \\
& E_{1}(\infty)=\min _{\psi \in \mathscr{F}_{0} \cap \operatorname{ker} H_{I}} \frac{\left\langle\psi\left|H_{\infty}\right| \psi\right\rangle}{\langle\psi \mid \psi\rangle} \leqq\left\langle\psi_{1}\left|H_{\infty}\right| \psi_{1}\right\rangle=\left\langle\psi_{1}\left|H_{U_{i}}\right| \psi_{1}\right\rangle
\end{aligned}
$$

and

$$
\begin{aligned}
E_{1}\left(U_{i}\right) & \leqq\left\langle\psi_{1}\left|H_{U_{i}}\right| \psi_{1}\right\rangle=\left\langle\psi_{1}\left|H_{0}\right| \psi_{1}\right\rangle \\
& =\left\langle\psi_{1}\left(U_{i}\right)\left|H_{0}\right| \psi_{1}\left(U_{i}\right)\right\rangle+\varepsilon_{i} \leqq E_{1}\left(U_{i}\right)+\varepsilon_{i},
\end{aligned}
$$


where we used Eq. (A.2). In (A.11), $\varepsilon_{i} \rightarrow 0$ as $i \rightarrow \infty$, thus

$$
E_{1}(\infty) \leqq\left\langle\psi_{1}\left|H_{\infty}\right| \psi_{1}\right\rangle=\bar{E}_{1} \text {. }
$$

From Eq. (A.8) we conclude that in Eq. (A.12) the equality holds. Since $E_{1}(\infty)$ is the minimal eigenvalue, $\psi_{1}$ must be an eigenstate. Now suppose we proved

$$
E_{k}(\infty)=\bar{E}_{k} \text { and } H_{\infty} \psi_{k}=\bar{E}_{k} \psi_{k}
$$

for $k=1, \ldots, n-1$. Then by the minimax principle

$$
\begin{aligned}
E_{n}(\infty) & =\min \left\{\left\langle\psi\left|H_{\infty}\right| \psi\right\rangle \mid \psi \in \mathscr{F}_{0} \cap \operatorname{ker} H_{I},\|\psi\|=1,\left\langle\psi \mid \psi_{k}\right\rangle=0, k=1, \ldots, n-1\right\} \\
& \leqq\left\langle\psi_{n}\left|H_{\infty}\right| \psi_{n}\right\rangle=\left\langle\psi_{n}\left|H_{0}\right| \psi_{n}\right\rangle \\
& =\left\langle\psi_{n}\left(U_{i}\right)\left|H_{0}\right| \psi_{n}\left(U_{i}\right)\right\rangle+\varepsilon_{i} \leqq E_{n}\left(U_{i}\right)+\varepsilon_{i}
\end{aligned}
$$

where $\varepsilon_{i} \rightarrow 0$, and thus

$$
E_{n}(\infty) \leqq\left\langle\psi_{n}\left|H_{\infty}\right| \psi_{n}\right\rangle \leqq \bar{E}_{n} .
$$

Due to Eq. (A.8) we have, in fact, equalities. Since $\psi_{n}$ is orthogonal to the first $n-1$ eigenstates of $H_{\infty}$, it must be an eigenstate.

As a corollary we obtain that Nagaoka's result extends to large but finite values of $U$. Indeed, he finds for $H_{\infty}$ a highest-spin ground state which (in $v>1$ dimensions) is not degenerated with eigenstates of lower spin. By continuity this remains valid down to some $U_{c}(\Lambda)$ which must diverge with the increasing volume at least as fast as $N L_{\max }^{2}$, as one can see from Eq. (3.24).

The above proposition can also be proved by a simple perturbation argument. I thank A. Mielke for this remark.

\section{A.2. One Dimensional Hubbard Model at $U=\infty$ with free Boundary Condition}

Proposition 3. Consider $H_{\infty}$ on the set $\Lambda=\{1,2, \ldots, L\}$ with free boundary condition and sign-keeping nearest-neighbor hopping $t_{x, x+1}$. For any $N<L, H_{\infty}$ has ground states with all possible values of $S$.

Proof. Lieb and Mattis [1] proved that in one dimension for hard core potential and with free boundary condition

$$
E_{\Lambda, N}(N / 2) \geqq E_{\Lambda, N}(N / 2-1) \geqq \cdots \geqq E_{\Lambda, N}\left(S_{\min }\right) .
$$

It suffices therefore to show that there is a ground state with $S=N / 2$. This is, however, elementary:

Suppose, e.g., $t_{x, x+1} \geqq 0$. One can construct an orthonormal basis in ker $H_{I}$ by choosing any ordered set $X \subset \Lambda$ of $N$ points, $X=\left(x_{1}<\cdots<x_{N}\right)$ and any set of $N$ spins $\Sigma=\left(\sigma_{1}, \ldots, \sigma_{N}\right)$ and defining

$$
|X, \Sigma\rangle=c_{x_{1} \sigma_{1}}^{\dagger} \cdots c_{x_{N} \sigma_{N}}^{\dagger}|0\rangle \text {. }
$$

Let $y \notin X$ and $1 \leqq j \leqq N$. Then

$$
\begin{aligned}
c_{y \sigma}^{\dagger} c_{x_{j} \sigma_{j}}|X, \Sigma\rangle & =\cdots c_{x_{j-1} \sigma_{j-1}}^{\dagger} c_{y \sigma}^{\dagger} c_{x_{j+1} \sigma_{j+1}}^{\dagger} \cdots|0\rangle \\
& =(-1)^{\text {no.of occupied sites between } x_{j} \text { and } y}\left|X^{\prime}, \Sigma^{\prime}\right\rangle,
\end{aligned}
$$


where $\left|X^{\prime}, \Sigma^{\prime}\right\rangle$ is another element of the basis. Since there is no occupied site between nearest neighbors, the nonvanishing matrix elements $\left\langle X^{\prime}, \Sigma^{\prime}\left|H_{\infty}\right| X, \Sigma\right\rangle$ are all negative. Let

$$
\psi=\sum A_{X, \Sigma}|X, \Sigma\rangle
$$

be any ground state of $H_{\infty}$. Then

$$
|\psi|=\sum\left|A_{X, \Sigma}\right||X, \Sigma\rangle
$$

is also a ground state, because

$$
\begin{aligned}
\left\langle|\psi|\left|H_{\infty}\right||\psi|\right\rangle & =\sum\left|A_{X, \Sigma} A_{X^{\prime}, \Sigma^{\prime}}\right|\left\langle X^{\prime}, \Sigma^{\prime}\left|H_{\infty}\right| X, \Sigma\right\rangle \\
& \leqq \sum A_{X, \Sigma} A_{X^{\prime}, \Sigma^{\prime}}\left\langle X^{\prime}, \Sigma^{\prime}\left|H_{\infty}\right| X, \Sigma\right\rangle \\
& =\left\langle\psi\left|H_{\infty}\right| \psi\right\rangle,
\end{aligned}
$$

and $\psi$ and $|\psi|$ have the same norm. We may suppose that $|\psi|$ is an eigenstate of $S^{z}$ with eigenvalue $M$. Then

$$
\left(S^{+}\right)^{N / 2-M}|\psi| \neq 0
$$

and hence $|\psi|$ is an $S=N / 2$ state. If $t_{x, x+1} \leqq 0$, we may either redefine the creation and annihilation operators by changing their sign for, say, any $x$ odd and then work with (A.16), or use the basis

$$
|X, \Sigma\rangle_{-}=(-1)^{x_{1}+\cdots+x_{N}}|X, \Sigma\rangle \text {. }
$$

In both cases $H_{\infty}$ will be represented by a matrix of $\leqq 0$ elements.

Remark. If the boundary condition is periodic (i.e., we admit $t_{1, L} \neq 0$ ) and $N$ is odd then between two nearest neighbor sites, the one of which is occupied and the other is empty, the number of occupied sites is even $(0$ or $N-1)$. Hence, (i) for $t_{x y} \geqq 0$ the matrix of $H_{\infty}$ in the basis (A.16) has non-positive elements and, (ii) for $t_{x y} \leqq 0$ and $L$ even, one finds a matrix with non-positive elements in the basis (A.19). We conclude that there are ground states with maximal spin. This is a new proof of an old result by Herring [13], reproduced also Aizenman and Lieb [5].

\section{References}

1. Lieb, E. H., Mattis, D.: Theory of ferromagnetism and ordering of electronic levels. Phys. Rev. 125, 164-172 (1962)

2. Lieb, E. H.: Two theorems on the Hubbard model. Phys. Rev. Lett. 62, 1201 (1989)

3. Nagaoka, Y.: Ferromagnetism in a narrow, almost half-filled $s$ band. Phys. Rev. 147, 392-405 (1966)

4. Tasaki, H.: Extension of Nagaoka's theorem on the large- $U$ Hubbard model. Phys. Rev. B40, 9192-9193 (1989)

5. Aizenman, M., Lieb, E. H.: Magnetic properties of some itinerant electron systems at $T>0$. Phys. Rev. Lett. 65, 1470 (1990)

6. Mielke, A.: The one-dimensional Hubbard model for large or infinite U. J. Stat. Phys. 62, 509-528 (1991)

7. Shastry, B. S., Krishnamurthy, H. R., Anderson, P. W.: Instability of the Nagaoka ferromagnetic state of the $U=\infty$ Hubbard model. Phys. Rev. B41, 2375-2379 (1990) 
8. Roth, L. M.: Spin wave stability of the ferromagnetic state for a narrow s-band. J. Phys. Chem. Solids 28, 1549-1555 (1967)

9. Maradudin, A. A., Montroll, E. W., Weiss, G. H., Ipatova, I. P.: Theory of lattice dynamics in the harmonic approximation, Ch.IV. New York: Academic Press 1971

10. Fang, Y., Ruckenstein, A. E., Dagotto, E., Schmitt-Rink, S.: Holes in the infinite- $U$ Hubbard model: Instability of the Nagaoka state. Phys. Rev. B40, 7406-7409 (1989)

11. Douçot, B., Wen, X. G.: Instability of the Nagaoka state with more than one hole. Phys. Rev. B40, 2719-2722 (1989)

12. Kato, T.: Perturbation theory for linear operators. Berlin, Heidelberg, New York: Springer 1966

13. Herring, C.: On the possibility of saturated ferromagnetism. Phys. Rev. B11, 2056-2061 (1975)

Communicated by J. Fröhlich 